\title{
Bronchiolitis obliterans mit organisierender Pneumonie (BOOP)
}

\author{
H. Teschler \\ T. E. Wessendorf
}

\section{Bronchiolitis Obliterans Organizing Pneumonia (BOOP)}

\section{Zusammenfassung}

Die Bronchiolitis obliterans mit organisierender Pneumonie (BOOP) ist eine Lungenerkrankung mit charakteristischen klinischen, radiologischen und histologischen Merkmalen. Der typische Patient stellt sich mit einer fieberhaften Erkrankung und fleckförmigen Verschattungen im Röntgenbild vor, die nicht auf Antibiotika angesprochen haben. Charakteristischerweise finden sich bei der BOOP Granulationsgewebspfröpfe, die das Lumen der terminalen und respiratorischen Bronchiolen ausfüllen und sich in die Alveolargänge vorschieben. Andere histologische Befunde beinhalten eine chronische interstitielle Entzündung und die vermehrte Ansammlung von Schaumzellen in den Alveolarräumen, so dass das histologische Muster einer organisierenden Pneumonie entsteht. Obwohl zunächst als kryptogen organisiernde Pneumonie beschrieben, bevorzugen Kliniker heutzutage die Bezeichnung BOOP. Am häufigsten kommt die idiopathische BOOP vor. Die rapid progressive und die noduläre BOOP sind insgesamt selten. Die BOOP wurde in das Spektrum pulmonaler Komplikationen bei verschiedenen Infektionen, im Zusammenhang mit Medikamenten, Bindegewebserkrankungen, Knochenmark-, Nieren- Lungentransplantation, sowie nach Bestrahlung von Brustkrebs aufgenommen. Bei Patienten mit typischem klinischen Bild und CT-Befund ist zur Bestätigung der Diagnose meist eine Bronchoskopie mit transbronchialer Biopsie und BAL ausreichend. Die BOOP spricht gewöhnlich gut auf eine Behandlung mit Kortikosteroiden an, rezidiviert aber in einem Drittel der Fälle. Bei sekundärer BOOP kann die Steigerung der täglichen Kortikosteroiddosis notwendig werden, doch ist gutes Ansprechen ebenfalls die Regel.

\section{Abstract}

Bronchiolitis obliterans organizing pneumonia (BOOP) is a lung disease with distinctive clinical, radiological and histological findings. The classical patient presents with a febrile illness and patchy infiltrates on chest x-ray who have not responded to antibiotics. BOOP is characterized by intraluminal granulation tissue polyps filling the lumens of terminal and respiratory bronchioles and extending into alveolar ducts. Other histological features include a chronic interstitial inflammation and the accumulation of foamy macrophages in the alveoli, representing an organizing pneumonia. Although initially described as cryptogenic organizing pneumonia (COP) the term BOOP is nowadays preferred by most clinicians. Idiopathic BOOP is the most frequent type. Rapid progressive and nodular BOOP are rare manifestations. BOOP has been added to the spectrum of pulmonary lesions associated with infections, different types of drugs, connective tissue disorders, bone marrow, renal, and lung transplantation and radiotherapy for breast cancer. In patients presenting with the classical clinical picture and CT pattern bronchoscopy with transbronchial biopsy and BAL is the method of choice for establishing the diagnosis. BOOP responds generally favourable to corticosteroid therapy but might recur in one third. The prognosis of primary BOOP is almost always excellent. In cases with secondary BOOP an increase of the daily dose of corticosteroids might be necessary but response is usually also favourable. 
Definition

In jüngster Zeit hat die Klassifikation der idiopathischen interstitiellen Pneumonien einen Wandel erfahren. Die von Katzenstein und Myers vorgelegte pathologisch-anatomische Einteilung ordnet der idiopathischen Lungenfibrose (IPF) als histopathologisches Substrat die „usual interstitial pneumonia = UIP“ nach Liebow zu. Davon werden einerseits die respiratorische Bronchiolitis-interstitielle Lungenerkrankung (RB-ILD) als Erkrankung überwiegend des Rauchers und als neue Entitäten die akute interstitielle Pneumonie (AIP) sowie die nicht spezifische interstitielle Pneumonie (NSIP) abgegrenzt $[2,4,30]$. Als fünfte histologische Erscheinungsform wird die kryptogen organisierende Pneumonie (engl. cryptogenic organizing pneumonia=COP) bzw. idiopathische Bronchiolitis obliterans mit organisierender Pneumonie (BOOP) genannt [17,21]. Diese Entitäten unterscheiden sich nicht nur hinsichtlich des pathologisch-anatomischen Korrelats, sondern auch bezüglich des klinischen Verlaufs und Ansprechens auf die Standardtherapie (Abb. 1) [10,32,47].

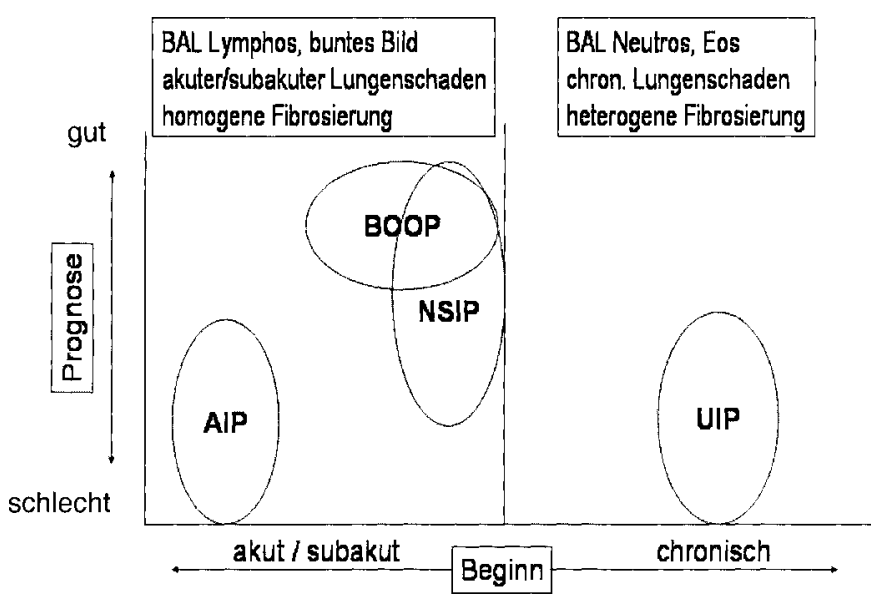

Abb. 1 Spektrum der idiopathischen interstitiellen Pneumonien (modifiziert nach [47]).

\section{Abgrenzung der BOOP}

Die Bronchiolitis obliterans mit organisierender Pneumonie (BOOP) wurde 1985 von Epler und Mitarbeitern als eigenständige Entität im Spektrum der interstitiellen Lungenerkrankungen charakterisiert [21]. Bereits ein Jahr zuvor hatte Davison das gleiche Krankheitsbild unter dem Namen „Kryptogen organisierende Pneumonie“ (engl. „cryptogenic organizing pneumonia = COP“) beschrieben [17]. Obwohl sich weltweit die Bezeichnung „BOOP“ durchzusetzen schien, wird neuerdings von internationalen Expertenkommissionen der Terminus „COP“ für die idiopathische Variante dieser Erkrankung bevorzugt [4,32]. Als Begründung wird die Tatsache genannt, dass Organisationsgewebspfröpfe in den Alveolen (organisierende Pneumonie) das vorherrschende histopathologische Substrat sind, während Bindegewebsproliferate in den Bronchiolen (obliterative Bronchiolitis) im Einzelfall fehlen können $[4,10,32]$. Außerdem bildet die organisierende Pneumonie - und nicht die Bronchiolitis - das röntgenologische Substrat der Erkrankung [17]. In Deutschland ist weiterhin BOOP die geläufigste Bezeichnung.
Die BOOP lässt sich klinisch, radiologisch und prognostisch eindeutig von der Atemwegserkrankung „obliterative Bronchiolitis“ (keine organisierende Pneumonie) und einer interstitiellen Lungenfibrose vom Typ der UIP/IPF unterscheiden [20,21,32]. Die BOOP ist histopathologisch gekennzeichnet durch eine Akkumulation von myxoidem, fibroblastenreichem Bindegewebe, das sich als Granulationsgewebe in den Alveolargängen ausbreitet und von dort kontinuierlich in die Bronchiolen vorschiebt (Abb. 2) [21]. Dieses pathologisch-anatomische Substrat ist nicht spezifisch für die BOOP, sondern ein entzündliches Reaktionsmuster der Lunge, das bei unterschiedlichen Schädigungsmechanismen als Reparationsprozess anzutreffen ist [10,32].

Andere histologische Merkmale beinhalten Cluster von mononukleären Entzündungszellen, die sich in den intraluminalen Granulationsgewebspfröpfen nachweisen lassen, eine chronische Entzündungsreaktion der umgebenden Alveolarwände, erkennbar an aktivierten Pneumozyten Typ II sowie einem dichten Lymphozyteninfiltrat und schaumige Makrophagen im Alveolarlumen $[10,21,32]$. Diese Form der organisierenden Pneumonie ist das pathologisch-anatomische Korrelat der COP als klinisch-radiologische Entität [17].

\section{Ätiologie der BOOP}

Der BOOP liegt keine spezifische Noxe zugrunde, vielmehr kommen zahlreiche Auslöser infrage [11,20]. Bei den meisten Patienten ist die Ursache der BOOP unbekannt. Von dieser idiopathischen Variante werden Fälle mit bekannter Ursache, beispielsweise Infektionen (Tab.1) sowie Systemerkrankungen mit einer BOOP als pulmonale Komplikation abgegrenzt [10,11,42]. Als Sonderform wird von diesen Varianten die fokale BOOP zumeist mit nodulären Verschattungen unterschieden. Das histologische

Tab. 1 Infektiöse Ursachen der BOOP (nach [11])

Bakterien
Chlamydia pneumoniae
Coxiella burnetii
Legionella pneumophila
Mycoplasma pneumoniae
Nocardia asteroides
Pseudomonas aeruginosa
Serratia marcescens
Staphylococcus aureus
Streptococcus Gruppe B
Streptococcus pneumoniae
Viren
Herpes simplex
Human immunodeficiency virus
Influenza
Parainfluenza
Parasiten
Plasmodium vivax
Pilze
Cryptococcus neoformans
Penicillium janthinellum
Pneumocystis carinii


Muster der BOOP wird außerdem bei Erkrankungen wie der UIP/ IPF, der Wegener'schen Granulomatose, im Randbereich von Lungenabszessen, in Nachbarschaft von malignen Lymphomen und anderen Tumoren der Lunge sowie bei Bronchiektasen beobachtet $[10,32,42]$. Bei diesen Patienten sind die geklagten Symptome meist Ausdruck der Grunderkrankung, die zudem den klinischen Verlauf bestimmt.

\section{Pathogenese der BOOP}

Die BOOP ist eine inflammatorische Lungenerkrankung, der primär ein entzündliches Reaktionsmuster der Alveolargänge und des peribronchialen Interstitiums zugrunde liegt, während die UIP/IPF primär durch einen Fibrosierungsprozess gekennzeichnet ist (Abb. 2) [10,32,47]. Wie im Frühstadium der UIP/IPF bestehen die Proliferate bei der BOOP aus einem frischen, fibromyxoiden Bindegewebe $[10,37,38]$. Während die Proliferate sich bei der BOOP nach Einleitung einer Kortikosteroidtherapie zurückbilden, schreitet der Umbauprozess bei UIP/IPF unaufhaltsam fort $[2,4,10,21]$. Die Ursachen für das unterschiedliche Ansprechen von BOOP und UIP/IPF auf die Gabe oraler Kortikosteroide ist unbekannt. Ein charakteristischer Unterschied zwischen beiden Entitäten ist die ausgeprägte Kapillarisierung der Granulationsgewebspfröpfe bei der BOOP im Vergleich zur UIP/ IPF [37]. Unter anderem wird vermutet, dass verschiedene Gefäßwachstumsfaktoren im Entzündungsprozess der BOOP eine normale Apoptoserate und damit einen ungestörten Zelltod ermöglichen, während dies bei der UIP/IPF nicht der Fall ist. Diese Annahme wird durch Untersuchungen gestützt, die im Granulationsgewebe der BOOP eine signifikant höhere Apoptoserate als bei UIP fanden [38]. Eine weitere Hypothese besagt, dass die Relation des Gewebsinhibitors für Metalloproteasen (TIMP) zu den Matrix-Metalloproteasen (MMPs) und die Konzentration der einzelnen Metalloproteinasen beim „remodelling“ des Granulationsgewebes und der interstitiellen Matrix eine zentrale Rolle spielt. Für das hohe Rückbildungspotenzial des Granulationsgewebes bei BOOP wird neuerdings die hohe kollagenolytische Aktivität von MMP-2 bezüglich Kollagen Typ IV in den Granulationsgewebspfröpfen verantwortlich gemacht [52].

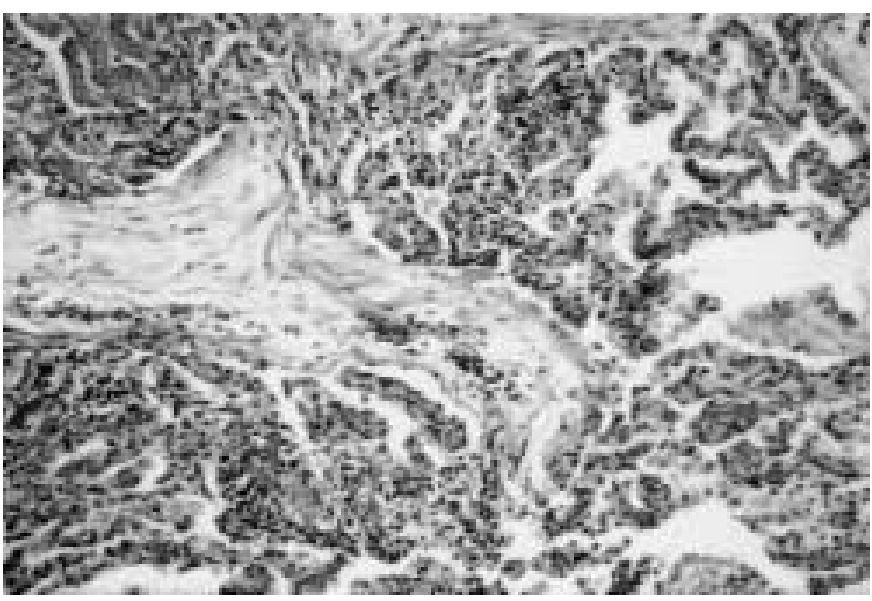

Abb. 2 Charakteristischer histologischer Befund einer BOOP. Im Zentrum findet sich ein fingerförmiger Granulationsgewebspfropf, der sich in den Alveolargängen und respiratorischen Bronchiolen ausbreitet. Ferner fällt ein Kollaps der nachgeschalteten Lufträume mit Schaumzellpneumonie im umgebenden Gewebe auf.

\section{Diagnostik der BOOP}

\section{Klinische Präsentation}

Bei BOOP sind Männer und Frauen etwa gleich häufig betroffen. Das typische Manifestationsalter beträgt 40-60 Jahre, doch kommt die Erkrankung auch bei Heranwachsenden und Greisen vor $[13-15,17,21,26]$. Prädisponierende Faktoren sind unbekannt. Insbesondere scheint keine Beziehung zum Raucherstatus zu existieren [21,26]. Die Erkrankung beginnt meist schleichend, seltener akut mit subfebrilen Temperaturen, trockenem Husten, Appetitlosigkeit und Gewichtsverlust (Tab.2). Nachtschweiß, Thoraxschmerzen, Bronchorrhö und Hämoptysen gehören nicht zum klassischen Bild der BOOP. Regelmäßig klagen die Patienten über Atemnot, die zumindest unter körperlicher Belastung auftritt. Typisch ist die schleichende Entwicklung der Symptomatik über mehrere Wochen, wobei das klinische Bild meist einer grippalen Erkrankung ähnelt. Nicht selten wird die Diagnose erst zwei bis drei Monate nach Wahrnehmung der ersten Symptome gestellt (Tab. 2) [42]. Bei der körperlichen Untersuchung findet sich auskultatorisch über den pulmonalen Infiltraten gelegentlich inspiratorisches Knisterrasseln. Bronchialatmen und obstruktive Nebengeräusche gehören nicht zum Spektrum der häufigen Befunde. Der Nachweis einer breiten Dämpfung ist selten, da ausgedehnte Pleuraergüsse eine Rarität darstellen. Das Vorhandensein von Uhrglasnägeln, insbesondere aber von Trommelschlägelfingern sollte Anlass sein, die Diagnose zu überdenken und eine fibrosierende oder maligne Lungenerkrankung in Erwägung zu ziehen.

\section{Bildgebende Diagnostik Röntgenthoraxbild}

Das charakteristische Röntgen-Thorax-Bild der BOOP zeigt fleckige, alveoläre Konsolidierungen, die bilateral angeordnet sind und die Lungenmittel- und -unterfelder bevorzugen (Abb.3). Zentrale Einschmelzungen sind selten und kommen überwie-

Tab. 2 Klinische Daten publizierter Serien inklusive der eigenen Patienten

\begin{tabular}{|c|c|c|c|}
\hline & $\begin{array}{l}\text { Epler [21] } \\
(n=112)\end{array}$ & $\begin{array}{l}\text { Costabel [15] } \\
(n=38)\end{array}$ & $\begin{array}{l}\text { Izumi [26] } \\
(n=34)\end{array}$ \\
\hline Männer/Frauen & $60 / 52$ & $17 / 21$ & $17 / 17$ \\
\hline mittleres Alter (Jahre) & $58(21-80)$ & $57(27-74)$ & $57(41-69)$ \\
\hline \multicolumn{4}{|l|}{ Raucherstatus } \\
\hline Nichtraucher & $43 \%$ & - & $56 \%$ \\
\hline Raucher & $25 \%$ & $26 \%$ & $26 \%$ \\
\hline Exraucher & $32 \%$ & - & $18 \%$ \\
\hline \multicolumn{4}{|l|}{ Symptome } \\
\hline Symptomdauer (Monate) & 3,6 & 5,5 & 7,5 \\
\hline Husten & $72 \%$ & $74 \%$ & $76 \%$ \\
\hline Fieber & $49 \%$ & $90 \%$ & $53 \%$ \\
\hline Atemnot & $49 \%$ & $90 \%$ & $47 \%$ \\
\hline \multicolumn{4}{|l|}{ physikalische Befunde } \\
\hline Knisterrasseln & $74 \%$ & $79 \%$ & $79 \%$ \\
\hline $\begin{array}{l}\text { Uhrglasnägel/Trommelschlä- } \\
\text { gelfinger }\end{array}$ & $5 \%$ & $5 \%$ & $3 \%$ \\
\hline \multicolumn{4}{|l|}{ pathologische Befunde } \\
\hline verminderte Vitalkapazität & $52 \%$ & - & $59 \%$ \\
\hline reduzierte Diffusionskapazität & $74 \%$ & - & $80 \%$ \\
\hline
\end{tabular}




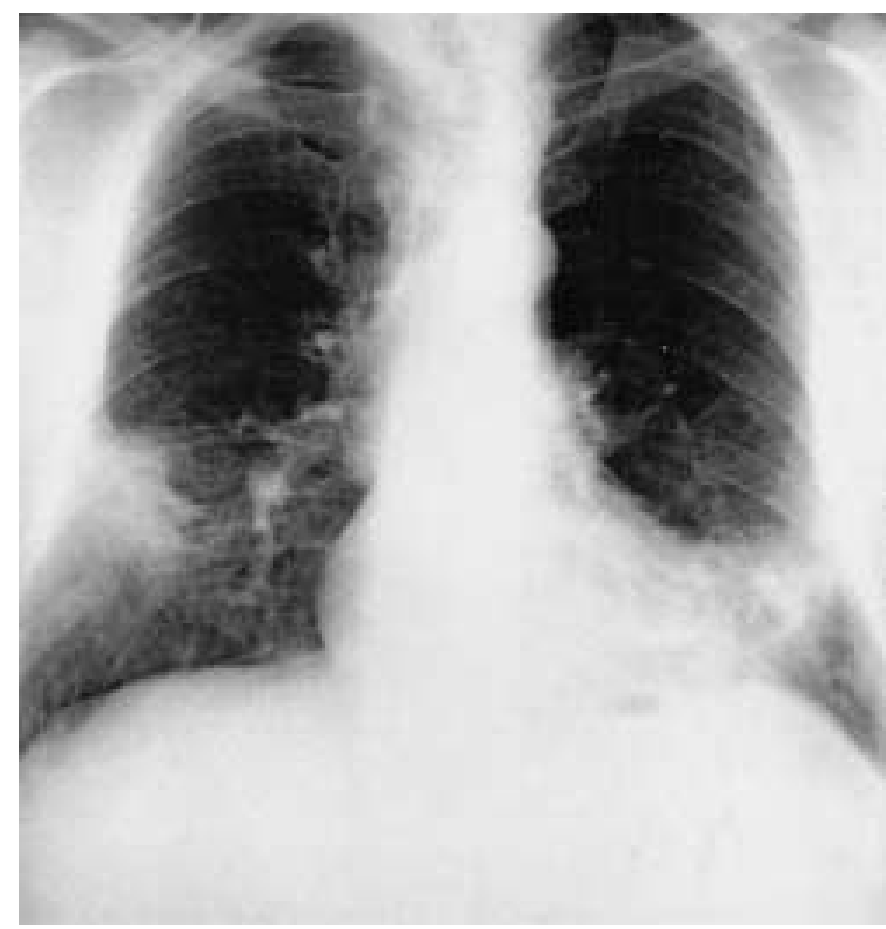

Abb. 3 Röntgenthoraxbild eines Patienten mit BOOP. Beachte die charakteristischen peripheren Infiltrate, die sich in den Lungenmittelund -unterfeldern darstellen.

gend bei der nodulären Variante (fokale BOOP) vor [46]. Vereinzelt wurden Pleuraergüsse zwar beschrieben, sollten aber stets Anlass zur differenzialdiagnostischen Abklärung sein. Unilaterale und wandernde Infiltrate kommen je nach Serie in bis zu 25\% vor $[13,31,34,49]$. Typisch für den progredienten Verlauf ist eine allmähliche Größenzunahme der Infiltrate, doch können an anderer Stelle auch neue Infiltrate hinzutreten.

\section{Thorax-CT}

Das charakteristische computertomographische Muster der BOOP ermöglicht dem erfahrenen Untersucher mit großer Sicherheit, in Kenntnis des klinischen Bildes, die korrekte Diagnose zu stellen [29]. Am häufigsten kommen milchglasartige Trübungen (90-100\%) und fleckige alveoläre Konsolidierungen (83-91\%) vor, die bilateral und peripher betont angeordnet sind (Abb. 4) $[29,44,45]$. Die Größe der Konsolidierungen variiert erheblich und reicht von wenigen Zentimetern bis hin zur Ausdehnung ganzer Segmente. Untersuchungen unserer Arbeitsgruppe führten zu der Erkenntnis, dass typische Konsolidierungen dreiecksförmig begrenzt sind, wobei die Spitze des Dreiecks hiluswärts orientiert ist und die Basis Pleurakontakt hat [15].

In einer HRCT-Studie von Murphy und Mitarbeitern wurden zwei typische Muster von linearen Verschattungen beschrieben: 1. peribronchial zentrierte Infiltrate mit Ausdehnung in Richtung der Pleura und 2. subpleurale Verdichtungszonen ohne klare anatomische Beziehung zum Bronchialbaum [45]. Beide Verschattungsmuster können multifokal und nebeneinander vorkommen, bevorzugen den Unterlappen und heilen binnen weniger Wochen nach Einleitung einer Kortikosteroidtherapie ohne Residuen ab [45].

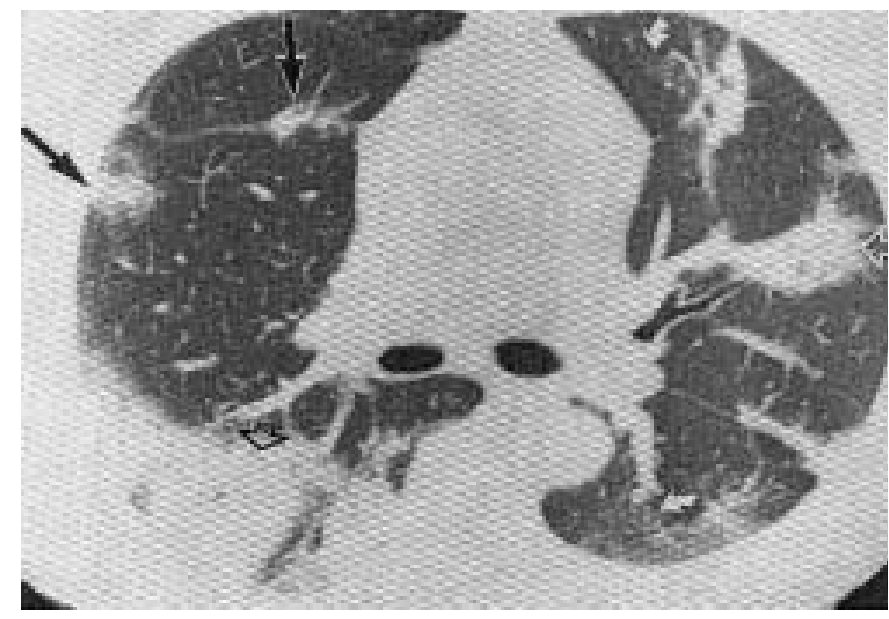

Abb. 4 BOOP im HRCT. Beidseits finden sich periphere Konsolidierungen, die zum Teil dreiecksförmig begrenzt sind (offener schwarzer Pfeil), daneben Bronchus-assoziierte Verschattungen (offener weißer Pfeil), flaue Infiltrate (dünne schwarze Pfeile) und milchglasartige Trübungen (gebogene weiße Pfeile).

Die beschriebenen CT-Muster sind zwar charakteristisch für die BOOP, nicht aber krankheitsspezifisch. Die Differenzialdiagnostik umfasst insbesondere das multilokuläre bronchoalveoläre Karzinom, niedrig maligne Lymphome mit Lungenbeteiligung, seltener die Alveolarproteinose, flüchtige eosinophile Infiltrate und die chronisch eosinophile Pneumonie und als Rarität emboliebedingte Lungeninfarkte.

Seltenere computertomographische Manifestationen der BOOP sind retikulonoduläre Verschattungen in Kombination mit milchglasartigen Trübungszonen, bei denen es sich histologisch zumeist um Organisationsstadien eines diffusen Alveolarschadens, um eine NSIP, seltener um eine AIP oder UIP/IPF mit Granulationsgewebspfröpfen als Reparationsprozess handelt $[29,44]$. Solitäre (fokale) BOOP-Läsionen mit entsprechendem radiologischen Muster finden sich gelegentlich auch im Randbereich von chronischen oder subakuten Entzündungsprozessen der Lunge. Die Diagnose wird durch chirurgische Lungenbiopsie oder nach kompletter Resektion des Herdes gestellt, die meist unter dem Verdacht auf ein Bronchialkarzinom oder einen anderen pulmonalen Tumor erfolgt $[10,46]$. Die solitären Herde sind oft im Oberlappen lokalisiert und wiesen in einer Serie in 4 von 5 Fällen eine zentrale Einschmelzung auf [46]. Recht seltene HRCT-Manifestationen der BOOP sind isolierte Areale mit milchglasartiger Trübung, multiple einschmelzende Knoten, zentral lokalisierte Verschattungen sowie ausgedehnte Pleuraergüsse.

Die prozentuale Häufigkeit der beschriebenen CT-Befunde bei BOOP ist in Tab. 3 zusammengestellt. Für Vergleichszwecke finden sich in dieser Tabelle auch Häufigkeitsangaben für die anderen idiopathischen interstitiellen Pneumonien.

\section{Lungenfunktion}

In der Lungenfunktion findet sich bei BOOP oft eine leichte bis mittelschwere Restriktion (Abb. 5) [15,17,21,26]. Eine obstruktive Komponente ist gewöhnlich nur bei langjährigen Zigarettenrauchern anzutreffen. Die arterielle Blutgasanalyse zeigt eine wechselnd ausgeprägte, zumeist leichte Hypoxämie, die unter 
Tab. 3 Häufigkeit typischer HRCT-Befunde bei BOOP und den anderen idiopathischen interstitiellen Pneumonien (nach [29])

\begin{tabular}{|c|c|c|c|c|c|}
\hline CT-Befunde & UIP (\%) & BOOP (\%) & DIP (\%) & $A I P(\%)$ & NSIP (\%) \\
\hline Milchglastrübung & 97 & 100 & 100 & 1000 & 100 \\
\hline $\begin{array}{l}\text { Atemwegskonsoli- } \\
\text { dierungen }\end{array}$ & 23 & 83 & 13 & 80 & 41 \\
\hline Honigwaben & 71 & 13 & 39 & 30 & 26 \\
\hline $\begin{array}{l}\text { intralobär retikuläre } \\
\text { Verdichtungen }\end{array}$ & 97 & 71 & 78 & 70 & 93 \\
\hline Noduli & 11 & 63 & 44 & 25 & 19 \\
\hline \multicolumn{6}{|l|}{ Befallsmuster } \\
\hline Oberlappen & 0 & 4 & 0 & 10 & 4 \\
\hline Unterlappen & 71 & 48 & 83 & 25 & 74 \\
\hline Ubiquitär & 29 & 48 & 17 & 65 & 22 \\
\hline \multicolumn{6}{|l|}{$\begin{array}{l}\text { anatomisches } \\
\text { Verteilungsmuster }\end{array}$} \\
\hline peripher & 89 & 71 & 43 & 10 & 85 \\
\hline peribronchovaskulär & 3 & 17 & 0 & 5 & 19 \\
\hline dorsal & 0 & 25 & 0 & 10 & 11 \\
\hline ubiquitär & 11 & 13 & 57 & 80 & 11 \\
\hline
\end{tabular}

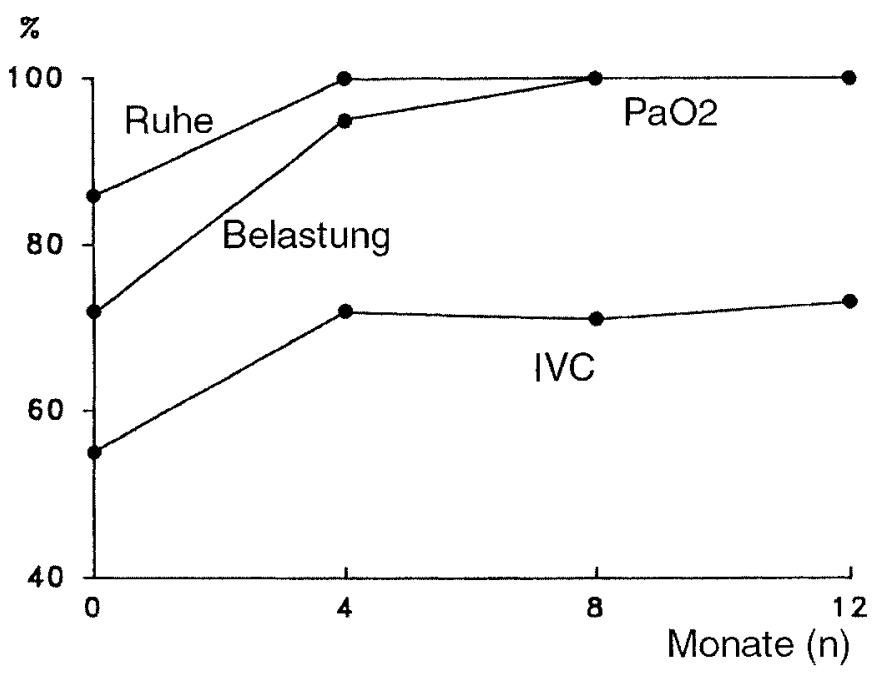

Abb. 5 Funktionsdaten unserer BOOP-Patienten zum Zeitpunkt der Diagnosestellung und im Verlauf nach Einleitung einer Kortikosteroidbehandlung (modifiziert nach [15]).

ergometrischer Belastung zunimmt [15]. Obwohl der Transferfaktor für Kohlenmonoxid schon eindeutig reduziert ist, kann der Transferkoeffizient noch normal sein. Bei schwerer Hypoxämie liegt ein ausgeprägter Rechts-Links-Shunt zugrunde, der Folge der organisierenden Pneumonie ist [11].

\section{Bronchoalveoläre Lavage}

Das Zellbild der bronchoalveolären Lavage (BAL) bei BOOP wird als „bunt“ beschrieben und ist gekennzeichnet durch eine obligate Vermehrung der Lymphozyten (20-40\%), meist in Kombination mit einer leichten Granulozytose $(<15 \%$ Neutrophilie, $<7 \%$ Eosinophilie) (Abb. 6) [13-15]. Als Besonderheiten sind in mehr als 50\% der Fälle Plasmazellen und Mastzellen bzw. schaumzellige Makrophagen nachweisbar. Immunzytologisch

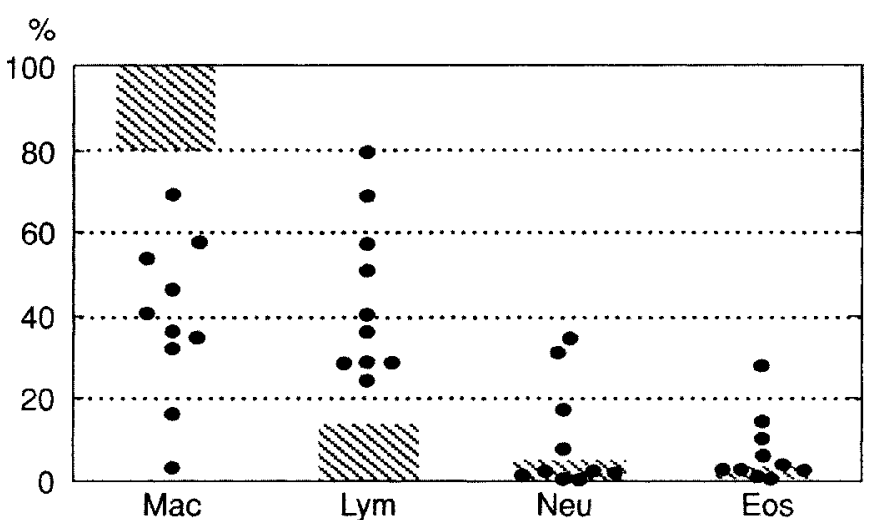

Abb. 6 BAL-Zellprofil bei BOOP (nach [14]).

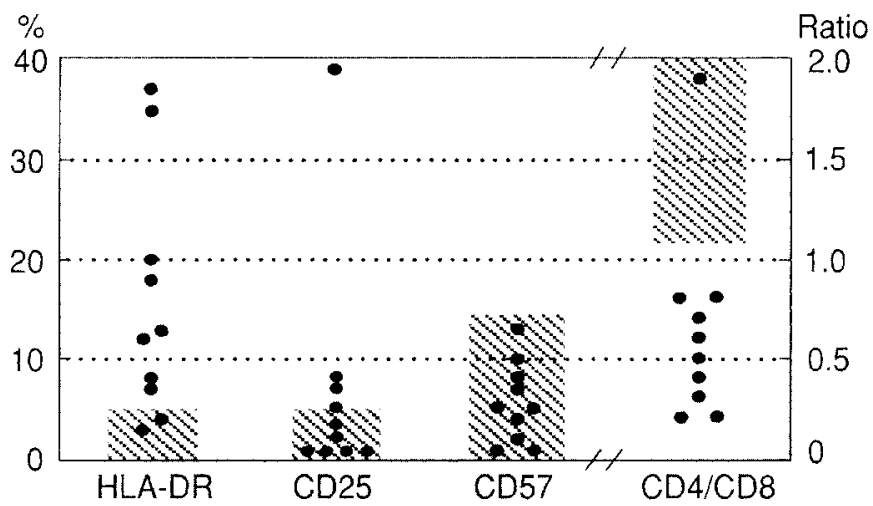

Abb. 7 Immunzytologisches Profil der BAL-Zellen bei BOOP (nach [14]).

ist der CD4/CD8-Quotient meist erniedrigt und die HLA-DR exprimierenden Zellen sind vermehrt, doch ist im Unterschied zur exogen allergischen Alveolitis, die ansonsten eine ähnliche Befundkonstellation aufweisen kann, der Prozentsatz der Leu7+ NK-Zellen nicht erhöht (Abb. 7) [15].

\section{Laborbefunde}

Für die BOOP existieren keine spezifischen Laborbefunde. Blutkörperchensenkungsgeschwindigkeit und C-reaktives Protein sind regelmäßig erhöht. Bei einem Drittel der Patienten finden sich BSG-Werte von über $60 \mathrm{~mm} / \mathrm{h}$. Bei $15-35 \%$ der Patienten besteht im Differenzialblutbild eine mäßige Leukozytose und in wechselndem Ausmaß eine Vermehrung der Neutrophilen $[11,42]$.

\section{Diagnosestellung}

Die Diagnose BOOP kann der erfahrene Untersucher mit großer Zuverlässigkeit bei Vorliegen der klinischen Trias aus grippeähnlicher Symptomatik, bilateral dreiecksförmig begrenzten, peripher angeordneten Konsolidierungen und einem BAL-Profil mit buntem Zellbild, leichter Lymphozytose und erniedrigtem CD4/CD8-Quotienten stellen [15,53]. In diesen lehrbuchmäßigen Fällen ist die Einleitung einer Kortikosteroidtherapie ohne die vorausgehende histologische Verifizierung der Diagnose gerechtfertigt, wenn eine engmaschige Verlaufskontrolle sichergestellt ist [15]. Ausbleibende Besserung oder ein Rezidiv trotz hoher Kortikosteroiddosen sollten den Verdacht auf eine andere Diagnose lenken und Anlass zur chirurgischen Lungenbiopsie ge- 
ben $[11,15,18,39]$. Geeignete Biopsiestellen sollten vorab mittels aktuellem HRCT lokalisiert werden, da es sich um fokale Veränderungen handelt und bei dieser Erkrankung in wenigen Tagen spontane Rückbildung möglich ist. Transbronchiale Biopsien in einer Sitzung in Kombination mit der BAL durchgeführt sind dann verwertbar, wenn die typischen bronchiolären und alveolären Veränderungen nebeneinander vorkommen.

\section{Behandlung der BOOP}

\section{Idiopathische BOOP}

Obwohl bei der BOOP Spontanremissionen bekannt sind, ist in den meisten Fällen die Indikation zur medikamentösen Behandlung gegeben. Orale Kortikosteroide sind Medikamente der Wahl $[11,13,15,17,21]$. Allerdings weichen die Angaben bezüglich Dosierung und Behandlungsdauer zum Teil erheblich voneinander ab. Während ältere Publikationen bei idiopathischer BOOP für ein bis zwei Monate die tägliche Gabe von 1,0 bis 1,5 mg Prednisolon pro Kilogramm Körpergewicht empfehlen, ist nach unseren Erfahrungen in Übereinstimmung mit aktuellen Behandlungsschemata anderer Arbeitsgruppen eine niedrigere Dosis von anfangs $40 \mathrm{mg}$ pro Tag bzw. von 0,5 bis $0,75 \mathrm{mg} / \mathrm{kg} / \mathrm{Tag}$ genauso wirksam - aber mit weniger Nebenwirkungen behaftet $[15,39]$. Die Anfangsdosis von $40 \mathrm{mg}$ pro Tag wird bei gutem Ansprechen schon nach 14 Tagen auf $30 \mathrm{mg}$ und anschließend stufenweise um $10 \mathrm{mg}$ pro Monat auf eine Erhaltungsdosis von 5-7,5 mg reduziert. Oft bessert sich das klinische Bild schon wenige Tage nach Behandlungsbeginn [17,21,42]. Im Mittel normalisiert sich das Röntgenthoraxbild nach 2 - 3 Monaten, die Lungenfunktion aber erst nach 3-6 Monaten [15]. Die Gesamtdauer der Behandlung beträgt zirka 6-12 Monate. Eine komplette und andauernde Remission ist die Regel $[39,42]$.

\section{Rezidivbehandlung}

Bei 15 bis $25 \%$ der Patienten kommt es ein- oder mehrfach zu einem Rezidiv [39,42]. Mit Rückfall ist insbesondere dann zu rechnen, wenn die Kortikosteroiddosis $15 \mathrm{mg} /$ Tag unterschreitet [39]. Das Risiko eines Rückfalls ist bei verzögertem Therapiebeginn erhöht. Multiple Rezidive finden sich gehäuft bei Patienten mit leichter Cholestase zum Zeitpunkt der Diagnosestellung [39]. Weder die Prognose der Erkrankung noch das erneute Ansprechen auf die Standardtherapie verschlechtert sich dadurch [39]. Zwar tritt das Rezidiv meist im anfangs befallenen Abschnitt auf, doch können andere Areale ebenfalls betroffen sein und neue hinzutreten. Bei unkomplizierten Rezidiven bietet eine aggressive Therapie gegenüber dem Standardschema keinen Vorteil, da die Komplikationsrate steigt, die Langzeiteffekte aber nicht besser sind als mit einer neuerlichen Anfangsdosis von beispielsweise $\leq 25 \mathrm{mg}$ pro Tag [39]. Die schrittweise Dosisreduktion erfolgt bei befriedigendem Ansprechen über einen Zeitraum von 6 bis 9 Monaten.

Nur selten ist der Übergang in eine chronische Verlaufsform zu befürchten, die vom Endstadium einer UIP/IPF nicht mehr zu unterscheiden ist. Bei diesen Patienten kann die kombinierte Gabe von Azathioprin oder Cyclophosphamid dennoch effektiv sein [9].
Tab. 4 Medikamente als Ursachen der BOOP (nach Referenz [11])

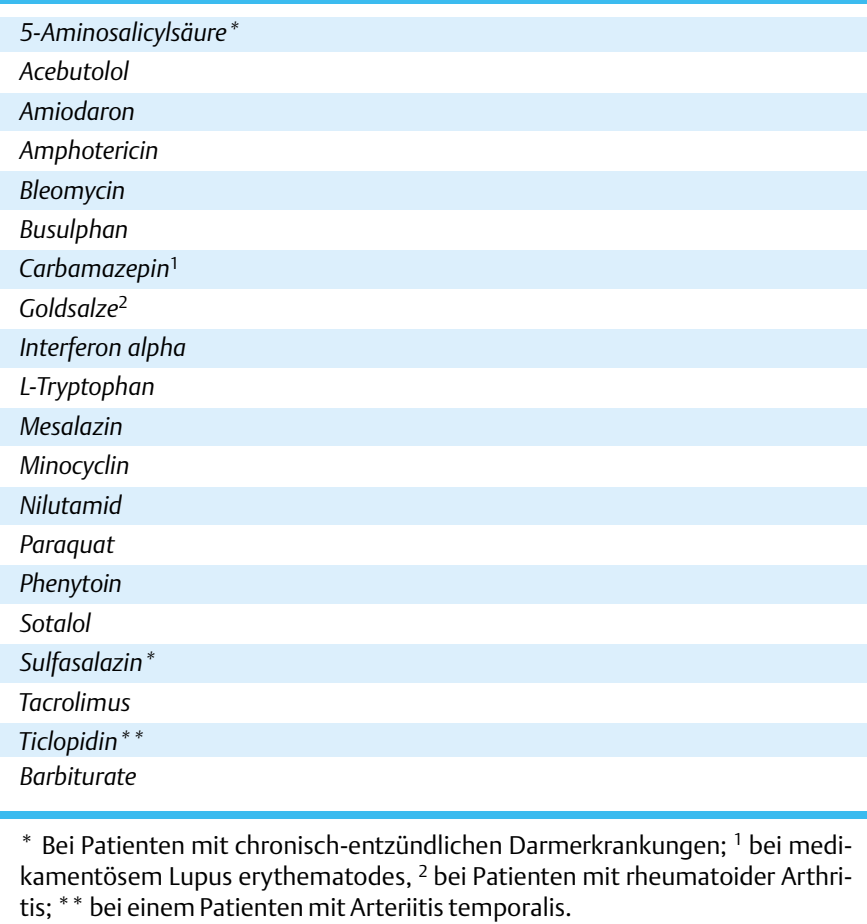

\section{Behandlung der sekundären und fokalen BOOP}

Bei Verdacht auf das Vorliegen einer BOOP als Reaktion auf Medikamente (siehe Tab. 4) steht der Auslassversuch an erster Stelle. Bei ausgeprägter Symptomatik wird parallel, sonst jedoch erst bei ausbleibender Besserung trotz Medikamentenentzug nach üblichem Schema behandelt.

Tritt die BOOP als Komplikation einer systemischen Erkrankung auf, wird primär die Grunderkrankung behandelt. Kortikosteroide werden hinzugefügt, wenn die Infiltrate sich nicht oder unvollständig zurückbilden. Höhere Kortikosteroiddosen von 100 bis $250 \mathrm{mg}$ pro Tag werden erforderlich, wenn Infiltrate unter laufender Steroidbehandlung der Systemerkrankung entstehen. Dies gilt auch bei Transplantationspatienten mit BOOP, die schon Kortikosteroide als Teil der Immunsuppression erhalten $[5,8,30,55]$. In all diesen Fällen wird stets zur histologischen Sicherung der Diagnose durch chirurgische Lungenbiopsie geraten.

Die Diagnose „fokale BOOP“ kann nur histologisch gestellt werden. Bei vollständiger Resektion eines solitären Herdes ist keine Nachbehandlung erforderlich. Selbst bei multiplen Knotenbildungen besteht eine hohe spontane Rückbildungsrate (70). Die Therapie nach Standardschema ist erst bei erkennbarer Progredienz oder bei ausgeprägter Symptomatik angezeigt (siehe unten).

\section{Besondere Formen}

\section{Rapid progrediente BOOP}

Bei wenigen Patienten mit BOOP wurde ein rapid progredienter Verlauf, zum Teil auch mit tödlichem Ausgang beschrieben [9,48]. Die Histologie entsprach bei einigen dieser Patienten 
dem Bild einer UIP mit ungewöhnlich stark ausgeprägter Granulationsgewebsbildung, während sich in anderen Fällen tatsächlich eine klassische, aber rasch progrediente BOOP zeigte [9]. Diese ungünstige Sonderform der BOOP findet sich gleichermaßen bei Männern und Frauen und kann Gesunde ebenso betreffen, wie Patienten mit Systemerkrankungen. Das klinische Bild kann dem eines ARDS mit rapidem Atemversagen innerhalb weniger Tage entsprechen, wobei histologisch dann häufig ein Mischbild zwischen diffusem Alveolarschaden und BOOP vorliegt. Klinisch ist die Unterscheidung zwischen akut progredienter BOOP und akuter interstitieller Pneumonie nicht möglich $[12,27]$. In entsprechenden Fällen wird deshalb frühzeitig zur histologischen Sicherung mittels offener Lungenbiopsie geraten, da die Einleitung einer hochdosierten Kortikosteroidtherapie die Prognose im Falle einer BOOP entscheidend verbessert, aber umgekehrt zu schweren Komplikationen führen kann, wenn es sich beispielsweise um eine nicht erkannte Pilzinfektion als weiterer Differenzialdiagnose handelt [48].

\section{Fokale BOOP}

1989 wurden in einer Serie von 16 Patienten mit gesicherter BOOP fünf Fälle mit fokal nodulärem Befallsmuster beschrieben [12]. Wichtigste Differenzialdiagnose zu dieser Variante der Erkrankung ist ein maligner Tumor bzw. eine pulmonale Metastasierung [46]. Obwohl sich aus den Noduli eine klassische BOOP entwickeln kann, ist dies eher die Ausnahme. In einer zweiten Serie wurden bei 12 Patienten multiple noduläre BOOP-Läsionen (2-8 mm) nachgesichert [1]. In allen Fällen bildete sich die klinische Symptomatik vollständig zurück, bei 10 Patienten spontan, bei 2 anderen nach Einleitung einer Kortikosteroidtherapie. Bemerkenswert ist die Tatsache, dass 50\% dieser Patienten über Pleuraschmerzen berichteten, die bei der typischen BOOP selten anzutreffen sind. Dass sich in den letzten Jahren kaum noch Berichte über die spontane Rückbildung von Lungenmetastasen in der Literatur finden, führen Chander und Mitarbeiter auf die heute übliche Durchführung einer offenen Lungenbiopsie zur histologischen Klärung zurück [7].

\section{Postinfektiöse BOOP}

Die postinfektiöse BOOP entwickelt sich bei einer breiten Palette von Pneumonien, unter anderem hervorgerufen durch Bakterien wie Chlamydia, Legionella und Mycoplasma pneumoniae, durch virale Erreger wie Parainfluenza- und Adenovirus, ferner durch Parasiten wie Plasmodien und durch Pilze unter Einschluss von Cryptococcus neoformans und Pneumocystis carinii $[6,19,28,35$, $41,56]$

Bei postinfektiöser BOOP bessert sich das pneumonische Infiltrat nach Einleitung einer gezielten antimikrobiellen Behandlung, doch bleibt ein Restinfiltrat bestehen, das oft an Dichte zunimmt. Dem radiologischen Korrelat entspricht eine organisierende Pneumonie mit Granulationsgewebspfröpfen in den betroffenen Alveolargängen und angrenzenden Bronchiolen. Im deutschen Schrifttum wird in dieser Situation von einer karnifizierenden Pneumonie gesprochen. Wenn sich die beschriebenen Veränderungen nach oraler Behandlung mit Kortikosteroiden in üblicher Dosis nicht binnen zwei bis drei Wochen zurückbilden, ist die histologische Sicherung des Prozesses anzustreben.

\section{BOOP als Medikamentennebenwirkung}

In den letzten Jahren wurden zahlreiche Fallberichte publiziert, die einen kausalen Zusammenhang zwischen Medikamenteneinnahme und Manifestation einer BOOP nahe legen. Unterschiedlichste Substanzklassen werden angeschuldigt: Antientzündlich, zytostatisch oder immunsuppressiv wirkende Medikamente, ferner Bluthochdruckmittel und Kardiaka sowie verschiedene Drogen, die missbräuchlich genommen werden (Tab. 4) [11]. Der entscheidende Schritt ist zunächst, an den Zusammenhang mit der Medikamenteneinnahme zu denken und dann die angeschuldigte Substanz konsequent abzusetzen. Die zusätzliche Behandlung mit Kortikosteroiden ist bei ausbleibender Besserung trotz Absetzens der vermuteten Noxe gerechtfertigt.

\section{BOOP bei Systemerkrankungen}

Klinisch unterscheidet sich die BOOP als Komplikation einer rheumatoiden Arthritis oder anderer Autoimmunerkrankungen des Bindegewebes kaum von der idiopathischen Verlaufsform. Es liegen Berichte über das Auftreten dieser Komplikation bei Patienten mit rheumatoider Arthritis, Lupus erythematodes, Dermatomyositis und Sjögren-Syndrom vor [22,24,36,51].

Während eine mit dieser Erkrankung assoziierte Lungenfibrose kaum auf Kortikosteroide anspricht, ist dies bei der hinzutretenden BOOP eher die Regel. Eine Ausnahme von dieser Regel scheint bei Dermatomyositis vorzuliegen, da sich die BOOP-typischen Veränderungen in einer publizierten Serie erst nach Gabe von Cyclophosphamid besserten [36]. Außerdem kommt die BOOP bei Morbus Bechterew, Polymyalgia rheumatica, Morbus Behçet, bei Immunmangelsyndromen und der essenziellen Kryoglobulinämie vor $[20,23,33]$.

\section{BOOP nach Organtransplantation}

Über die Entwicklung einer BOOP nach Transplantation von Knochenmark, Lunge beziehungsweise Niere wurde ebenfalls berichtet. Im Zusammenhang mit der Knochenmarktransplantation beschränken sich die vorliegenden Einzelberichte auf Patienten mit allogener oder syngener Transplantation, wobei es sich scheinbar gehäuft um das Zusammentreffen mit einer Zytomegalievirusinfektion handelt $[5,30]$. Ferner kommt die BOOP bei $10-28 \%$ der Patienten nach Lungentransplantation vor, wobei das zeitliche Zusammentreffen mit einer Abstoßungsreaktion möglich ist [8]. Vorliegende Berichte beziehen sich auf das erste Jahr nach Transplantation. Zur Behandlung werden meist höhere Kortikosteroiddosen eingesetzt. Stets muss eine Abstoßungsreaktion oder Infektion mit Problemkeimen ausgeschlossen und bei Bestätigung aggressiv behandelt werden [8]. Offen ist noch, ob die Manifestation einer BOOP als Risikofaktor für das Auftreten einer obliterativen Bronchiolitis - und damit einer chronischen Abstoßungsreaktion - zu werten ist. Über das Auftreten einer BOOP als Komplikation nach Nierentransplantation liegen nur wenige Berichte vor, die für eine schnelle Rückbildung der Veränderungen nach Einleitung oder Steigerung der täglichen Kortikosteroiddosis sprechen [55].

\section{BOOP nach Strahlentherapie}

In letzter Zeit haben mehrere Autoren über das gehäufte Auftreten und einige Besonderheiten der BOOP im Zusammenhang mit der Strahlentherapie bei an Brustkrebs erkrankten Frauen publi- 
ziert $[3,16,43,54]$. Die Erkrankung tritt 1 bis 18 Monate nach Abschluss der Strahlentherapie auf. In der bildgebenden Diagnostik dominieren fleckförmige, seltener auch diffuse mikronoduläre Verschattungen. Ein disseminiertes Verschattungsmuster ist insgesamt selten, kommt aber bei kleinherdigen, peribronchialen BOOP-artigen Läsionen auch ohne vorausgehende Strahlentherapie vor [40]. Die BOOP entwickelt sich häufig außerhalb des Strahlenfeldes und kann von der bestrahlten auf die nicht bestrahlte Lunge übergreifen. Diese sekundäre Form der BOOP spricht exzellent auf orale Kortikosteroide an, doch muss ebenfalls mit Rezidiven gerechnet werden.

\section{Weitere Ursachen einer BOOP}

Weitere Berichte liegen über die Entstehung einer BOOP durch Umwelteinflüsse und im Zusammenhang mit Arbeitsunfällen vor. Angeschuldigt werden beispielsweise die Einatmung von toxischen Aerosolen, Farbpartikeln und Penicilliumsporen sowie die Rauchgasinhalation bei Hausbränden [20]. Die Liste sonstiger Ursachen ist lang und beinhaltet das myelodysplastische Syndrom, die chronische Thyreoiditis, die alkoholische und primär biliäre Leberzirrhose, die HIV-Infektion, chronisch entzündliche Darmerkrankungen, maligne Lymphome und die T-Zell-Leukämie $[11,20]$.

\section{Prognose}

Die Prognose der primären und fokalen BOOP ist trotz möglicher Rezidive gut. So lebten fünf Jahre nach Diagnosestellung noch mehr als $80 \%$ der Erkrankten. Bei der sekundären BOOP hängt die Prognose maßgeblich von der Grunderkrankung ab. In der Serie von Lohr [42] lebten nach 5 Jahren nur noch 45\% der Patienten.

\section{Literatur}

${ }^{1}$ Akira M, Yamamoto S, Sakatani M. Bronchiolitis obliterans organizing pneumonia manifesting as multiple large nodules or masses. Am J Roentgenol 1998; 170: 291 - 295

${ }^{2}$ American Thoracic Society and European Respiratory Society. Idiopathic pulmonary fibrosis: diagnosis and treatment. Am J Respir Crit Care Med 2000; 161: 646-664

${ }^{3}$ Arbetter KR, Prakash UBS, Tazelaar HD et al. Radiation-induced pneumonitis in the „nonirradiated“ lung. Mayo Clin Proc 1999; 74: 27-36

${ }^{4}$ ATS/ERS. International multidisciplinary consensus classification of idiopathic interstitial pneumonias. (in Vorbereitung)

${ }^{5}$ Baron FA, Hermanne JP, Dowlati A et al. Bronchiolitis obliterans organizing pneumonia and ulcerative colitis after allogeneic bone marrow transplantation. Bone Marrow Transplant 1998; 21: 951 - 954

${ }^{6}$ Carey CR, Mueller L, Fotopoulos CL et al. Bronchiolitis obliterans organizing pneumonia associated with Cryptococcus neoformans infection (letter). Rev Infect Dis 1991; 13: 1253 - 1254

${ }^{7}$ Chander K, Feldman L, Mahajan R. Spontaneous regression of lung metastases: possible BOOP connection (letter)? Chest 1999; 115: $601-602$

${ }^{8}$ Chaparro C, Chamberlain D, Maurer J et al. Bronchiolitis obliterans organizing pneumonia (BOOP) in lung transplant recipients. Chest 1996; 1100: $1150-1154$

${ }^{9}$ Cohen AJ, King TE, Downey GP. Rapidly progressive bronchiolitis obliterans with organizing pneumonia. Intern Med 1994; 149: $1670-1675$

${ }^{10}$ Colby TV. Bronchiolitis. Am J Clin Pathol 1998; 109: 101 - 109

${ }^{11}$ Cordier JF. Organising pneumonia. Thorax 2000; 55: 318-328

12 Cordier JF, Loire R, Brune J. Idiopathic bronchiolitis obliterans organizing pneumonia: definition of characteristic clinical profiles in a series of 16 patients. Chest 1989; 96: 999-1004
${ }^{13}$ Costabel U, Guzman J, Teschler H. Bronchiolitis obliterans with organizing pneumonia: outcome. Thorax 1995; 50: S59-S64

${ }^{14}$ Costabel U, Teschler H, Guzman J. Bronchiolitis obliterans organizing pneumonia (BOOP): the cytological and immunocytological profile of bronchoalveolar lavage. Eur Respir J 1992; 5: 791 - 797

${ }^{15}$ Costabel U, Teschler H, Schoenfeld B et al. BOOP in Europe. Chest 1992; 102: 14 S-20S

${ }^{16}$ Crestani B, Valeyre D, Roden S et al. Bronchiolitis obliterans organizing pneumonia syndrome primed by radiation therapy to the breast. Am J Respir Crit Care Med 1998; 158: 1929-1935

17 Davison AG, Heard BE, Turner-Warwick WAC. Cryptogenic organizing pneumonitis. QJ M 1983; 52: 382 - 393

18 Deshmukh SP, Krasna MJ, Mclaughlin JS. Video assisted thoracoscopic biopsy for interstitial lung disease. Int Surg 1996; 81: 330-332

${ }^{19}$ Diehl JL, Gisselbrecht M, Meyer G et al. Bronchiolitis obliterans organizing pneumonia associated with chlamydial infection. Eur Respir J 1996; 9: 1320 - 1322

${ }^{20}$ Epler GE. Bronchiolitis obliterans organizing pneumonia. Arch Intern Med 2001; 161: 158-164

${ }^{21}$ Epler GR, Colby TV, Mcloud TC et al. Bronchiolitis obliterans organizing pneumonia. N Engl J Med 1985; 312 s: 152 - 158

22 Epler GR, Mark EJ. A 65-year-old woman with bilateral pulmonary infiltrates. N Engl J Med 1986; 314: 1627-1635

${ }^{23} \mathrm{Gul} \mathrm{A}$, Yilmazbayhan D, Buyukbabani $\mathrm{N}$ et al. Organizing pneumonia associated with pulmonary artery aneurysms in Behçet's disease. Rheumatology 1999; 38: 1285-1289

24 Imasaki T, Yoshi A, Tanaka S et al. Polymyositis and Sjögren's syndrome associated with bronchiolitis obliterans organizing pneumonia. Intern Med 1996; 35: 231-235

${ }^{25}$ Ippolito JA, Palmer L, Spector S et al. Bronchiolitis obliterans organizing pneumonia and rheumatoid arthritis. Semin Arthritis Rheum 1993; $23: 70-78$

${ }^{26}$ Izumi T. The global view of idiopathic bronchiolitis obliterans organizing pneumonia. In: Epler GR (ed). Diseases of the bronchiols. New York: Raven Press, 1994: 307-312

${ }^{27}$ Janz TG, Madan R, Marini JJ et al. Clinical conference on management dilemmas: progressive infiltrates and respiratory failure. Chest 2000; 117: $562-572$

28 Jeon JS, Yi HA, Ki SY et al. A case of bronchiolitis obliterans organizing pneumonia associated with adenovirus. Korean J Intern Med 1997; 12: $70-74$

29 Johkoh T, Muller NL, Cartier Y et al. Idiopathic interstitial pneumonias: diagnostic accuracy of thin-section CT in 129 patients. Radiology 1999; 211: 555-560

${ }^{30}$ Kanda Y, Takahashi T, Imai Y et al. Bronchiolitis obliterans organizing pneumonia after syngenic bone marrow transplantation for acute lymphoblastic leukaemia. Bone Marrow Transplant 1997; 19: $1251-1253$

${ }^{31}$ Kanwar BA, Shehan CJ, Campbell JC et al. A case of unilateral bronchiolitis obliterans organizing pneumonia. Nebr Med J 1996; 5: 149-151

${ }^{32}$ Katzenstein AL, Myers JL. Nonspecific interstitial pneumonia and the other idiopathic interstitial pneumonias: classification and diagnostic criteria. Am J Surg Pathol 2000; 24: 1-3

${ }^{33}$ Kaufman J, Komorowski R. Bronchiolitis obliterans organizing pneumonia in common variable immunodeficiency syndrome. Chest 1991; 100: $552-553$

${ }^{34}$ King TE. BOOP: an important cause of migratory pulmonary infiltrates? Eur Respir J 1995; 8: 193 - 195

35 Kleindienst R, Fend F, Prior C et al. Bronchiolitis obliterans organizing pneumonia associated with Pneumocystis carinii infection in a liver transplant patient receiving tacrolimus. Clin Transplant 1999; 13: 65-67

${ }^{36}$ Knoell KA, Hook M, Grice DP et al. Dermatomyositis associated with bronchiolitis obliterans organizing pneumonia (BOOP). J Am Acta Dermatol 1999; 40: 328 - 330

${ }^{37}$ Lappi-Blanco E, Kaarteenaho-Wilk R, Soini Y et al. Intraluminal fibromyxoid lesions in bronchiolitis obliterans organizing pneumonia are highly capillarized. Hum Pathol 1999; 30: $1192-1196$

${ }^{38}$ Lappi-Blanco E, Soini Y, Paakko P. Apoptotic activity is increased in the newly formed fibromyxoid connective tissue in bronchiolitis obliterans organizing pneumonia. Lung 1999; 177: 367-376

${ }^{39}$ Lazor R, Vandevenne A, Pelletier A et al. Cryptogenic organizing pneumonia. Characteristics of relapses in a series of 48 patients. Am J Respir Crit Care Med 2000; 162: 571-577 
${ }^{40}$ Lemm V, Sohrab S, Theegarten D et al. Bronchiolitis obliterans mit peribronchialer organisierender Pneumonie. Pneumologie 2001; 55: S65

${ }^{41}$ Llibre JM, Urban A, Garcia E et al. Bronchiolitis obliterans organizing pneumonia associated with acute Mycoplasma pneumoniae infection. Clin Infect Dis 1997; 25: 1340-1342

${ }^{42}$ Lohr RL, Boland BJ, Douglas WW et al. Organizing pneumonia. Arch Intern Med 1997; 157: $1323-1329$

${ }^{43}$ Majori M, Poletti V, Curti A et al. Bronchoalveolar lavage in bronchiolitis obliterans organizing pneumonia primed by radiation therapy to the breast. J Allergy Clin Immunol 2000; 105: 239-244

${ }^{44}$ Mihara N, Johkoh T, Ichikado K et al. Can acute interstitial pneumonia be differentiated from bronchiolitis obliterans organizing pneumonia by high-resolution CT? Radiat Med 2000; 18: 299-304

${ }^{45}$ Murphy JM, Schnyder P, Verschakelen J et al. Linear opacities on HRCT in bronchiolitis obliterans organizing pneumonia. Eur Radiol 1999; 9: $1813-1817$

${ }^{46}$ Murphy J, Schnyder P, Herold C et al. Bronchiolitis obliterans organizing pneumonia simulating bronchial carcinoma. Eur Radiol 1998; 8: $1165-1169$

${ }^{47}$ Nagai S, Kitaichi M, Izumi T. Classification and recent advances in idiopathic interstitial pneumonia. Curr Opin Pulm Med 1998; 4: 256-260

${ }^{48}$ Nizami IY, Kissner DG, Visscher DW et al. Idiopathic bronchiolitis obliterans with organizing pneumonia: an acute and life-threatening syndrome. Chest 1995; 108: $271-277$

${ }^{49}$ Peramaki E, Salmi L, Kava T et al. Unilateral bronchiolitis obliterans organizing pneumonia and bronchoalveolar lavage neutrophilia in a patient with parainfluenza 3 virus infection. Respir Med 1991; 85: $159-161$
${ }^{50}$ Siddiqui MT, Garrity ER, Husain AN. Bronchiolitis obliterans organizing pneumonia-like reactions. Hum Pathol 1996; 27: 714 - 719

${ }^{51}$ Stey C, Truninger K, Marti D et al. Bronchiolitis obliterans organizing pneumonia associated with polymyalgia rheumatica. Eur Respir J 1999; 13: $926-929$

52 Suga M, lyonaga K, Okamoto T et al. Characteristic elevation of matrix metalloproteinase activity in idiopathic interstitial pneumonias. Am J Respir Crit Care Med 2000; 162: 1949-1956

53 Teschler H, Costabel U, Greschuchna D et al. Bronchioliltis obliterans mit organisierender Pneumonie. Atemw Lungenkrkh 1989; 15: $288-292$

${ }^{54}$ van Laar JM, Holscher HC, van Krieken JHJM et al. Bronchiolitis obliterans organizing pneumonia after adjuvant radiotherapy for breast carcinoma. Respir Med 1997; 91: 241 - 244

55 Verberckmoes R, Verbeken E, Verschakelen J et al. BOOP after renal transplantation. Nephrol Dial Transplant 1996; 11: 1862 - 1863

${ }^{56}$ Yale SH, Adlakha A, Sebo TJ et al. Bronchiolitis obliterans organizing pneumonia caused by Plasmodium Vivax malaria. Chest 1993; 104: $1294-1296$

\section{Bereits publizierte Beiträge zu dieser Serie:}

${ }^{1}$ Pulmonale Lymphangioleiomyomatose. Pneumologie 2002; 56: $309-315$

${ }^{2}$ Alveolarproteinose. Pneumologie 2002; 56: $448-456$

${ }^{3}$ Idiopathische eosinophile Pneumonien. Pneumologie 2002; 56: 621-630 